\title{
Binding Surfaces and Structural Phase Transformations
}

\author{
M. Schröter, E. Hoffmann, M.S. Yang, P. Entel, H. Akai* and A. Altrogge \\ Theoretische Tieftemperaturphysik, Gerhard-Mercator-Universität, Gesamthochschule Duiburg, \\ 47048 Duisburg, Germany \\ * Department of Physics, Naura Medical University, Kashihara, Nara 634, Japan
}

\begin{abstract}
We have calculated binding surfaces for $c / a$ changes (along the Bain path) and changes of the atomic volume of metallic systems undergoing martensitic transformations. We find characteristic differences between the surfaces of nonmagnetic systems (with a close packed low temperature structure) and magnetic systems (with a less close packed low temperature structure). The ab initio calculations have been performed by using the augmentedspherical-wave method within the local-density approximation in the case of stoichiometrically ordered compounds and the Korringa-Kohn-Rostocker method together with the coherent-potential approximation in the case of disordered alloys.
\end{abstract}

\section{Introduction}

Many transition metals and their alloys have a rich phase diagram and some of them favor complex or even exotic lattice structures. The partially open d-bands with various degrees of valence band filling induce a variety of electronic behavior. A considerable part of these systems is magnetic or tends to be magnetic. Thus a zoo of elementary excitations may shape the low temperature behaviour.

With the tool of total energy band structure calculations and the feasability of binding surfaces one has the possibility to discuss not only ground state properties but also the influence of fluctuations and thus in principle of temperature. Computational and labour technical limitations so far restrict our ambitions to the structural simpler of these systems, still allowing to investigate the phase transformation between fcc and bcc structure and the interplay between the structural phase transformation and magnetism.

\section{Methods}

Traditionally band structure calculations were designed to give insight into the relative position of energy bands and the density of electronic states. With the local-density approximation (LDA) [1] total energy calculations became possible, allowing the calculation of binding curves, lattice constants and magnetic moments of solids[2]. With the capacity of modern computers and improved algorithms, binding surfaces make more detailed information about ground state properties available[3,4]. Moreover, since these binding surfaces represent a cross section of the phase space of the system, theoretical insight into the thermodynamical behavior can be gained[5].

For ordered structures the augmented-sperical-wave method by Williams, Kübler and Gelatt [6], which uses a small and efficient basis set for the wave functions and the so called atomic-sphere approximation, is well known and tested [2,3]. In our implemetation we use the von Barth-Hedin exchange functional [7] with a modification by Janak [7] in a non relativistic version. Recent results obtained with this method concern the relation between electronic structure and magnetovolume instabilities of Invar alloys and the laves phase compound $\mathrm{Fe}_{2} \mathrm{Ti}[9]$

The Korringa-Kohn-Rostocker (KKR) method [10] for band structure calculations relies on a Greens function formalism. Thus it can be combined with the coherent-potential approximation [11] and allows the investigation of alloys. Apparently, together with the LDA, it is a very powerful method.[12] In our 
implementation we use the muffin-tin approximation and a scalar relativistic approximation. A side effect of the scalar relativistic approximation is to give too small equilibrium volumes.
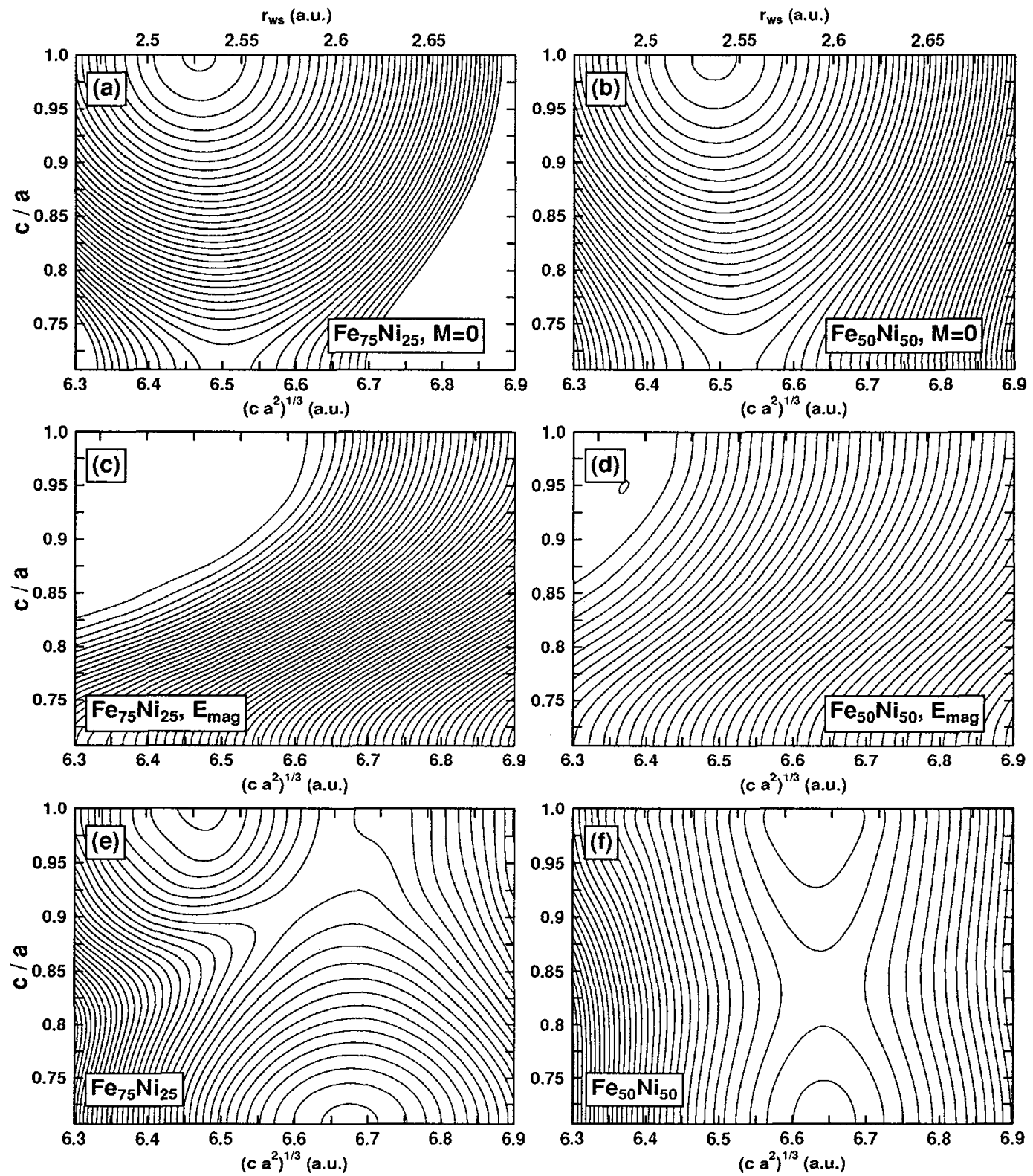

Figure 1 Contours of the total energy over the fcc lattice constant and the c/a ratio for nonmagnetic a) $\mathrm{Fe}_{75} \mathrm{Ni} 25$ and b) $\mathrm{Fe}_{50} \mathrm{Ni}$ (50, the magnetic contribution to the total energy of c) $\mathrm{Fe}_{75} \mathrm{Ni}_{25}$ and d) $\mathrm{Fe}_{50} \mathrm{Ni} 50$ and the resulting total energy of e) $\mathrm{Fe}_{75} \mathrm{Ni}_{25}$ and $\left.\mathrm{f}\right) \mathrm{Fe}_{50} \mathrm{Ni}_{50}$. Contour lines are in width of $0.5 \mathrm{mRy}$. The c/a ratio ranges from 0.7071 for the bcc-structure to 1 for the fcc-structure. A vertical cross section represents the Bain path from fcc to bcc at a constant volume. The line of zero magnetic contribution to the total energy is the first line to be seen coming from the upper left of Figure $1 \mathrm{c}$ and $1 \mathrm{~d}$. The magnetic energy increases with increasing volume. 


\section{Results}

\subsection{The Fe-Ni system}

In order to investigate the interplay between the structural phase transformation and the magnetic phase transition in Fe-Ni systems, we calculated magnetic and nonmagnetic structural binding surfaces, which are shown in Figure 1. The comparison between magnetic and nonmagnetic binding surface gives the magnetic contribution to the total enegy. Typically, if constrained to be nonmagnetic, these systems strongly favor the fcc structure. This tendency is the more pronounced the higher the Fe concentration. For pure nonmagnetic iron the energy difference between the fcc state and the bcc state is known to be $\approx 20 \mathrm{mRy}$, extrapolation of our data shows that this energy difference should not reverse sign for any considerable iron content. Magnetism on the other hand favors large volumes, in accordance with the Pauli principle. As can be seen in Figure 1c and 1d, this preference is weaker in the fcc structure, the energy difference between fcc and bcc structure on behalf of the magnetism is again the stronger the higher the Fe content. Further on the volume at which the magnetic moment breaks down for the fcc structure becomes larger with increasing $\mathrm{Fe}$ content, as can be seen from the line of zero energy for the magnetic contribution. This suggests that in the close packed fcc structure the system is further away from the atomic limit than in the less close packed bcc structure. The resulting structural binding surfaces (Figure le and 1f) show that for 50 at $\%$ iron the system is clearly magnetic and the energy difference between the fcc and bcc state is slightly in favor of the fcc state. With increasing iron percentage on the one hand the magnetic contributions for the bcc state become larger but also the volume at which the magnetic moment in the fcc state breaks down increases, thus reducing the magnetic influence in the fcc state and resulting in a metastable non magnetic fcc state beginning at 25 at\% iron, the energy barrier between the two states being approximately $7 \mathrm{mRy}$. For pure iron our KKR-CPA calculations result in a bcc magnetic ground state, which is by 3 mRy more stable than the nonmagnetic metastable fcc state, the energy barrier between them being approximately $12 \mathrm{mRy}$.

As previous investigations [13] show, the influence of disorder in the Fe-Ni system is to quench magnetism. This may be related to the fact that in the disordered system on the average the Fe-Fe nearest neighbour coordination is higher than in the ordered system and that the stability of the Fe moment is sensitive towards this coordination.

a) $\mathrm{Fe}-\mathrm{Ni}$

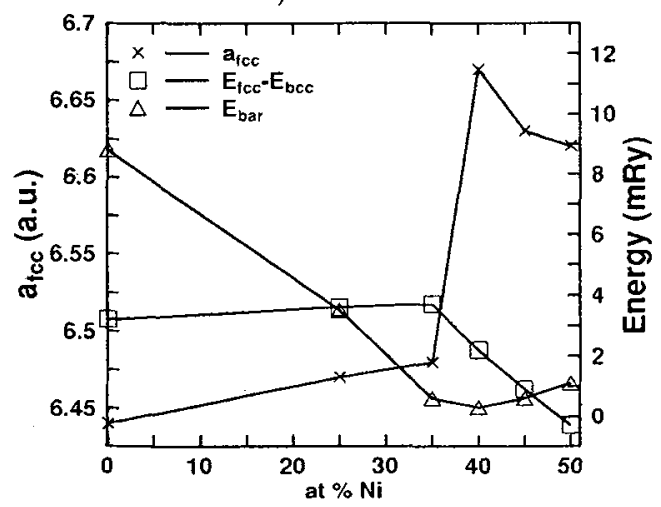

b) Cu-Zn

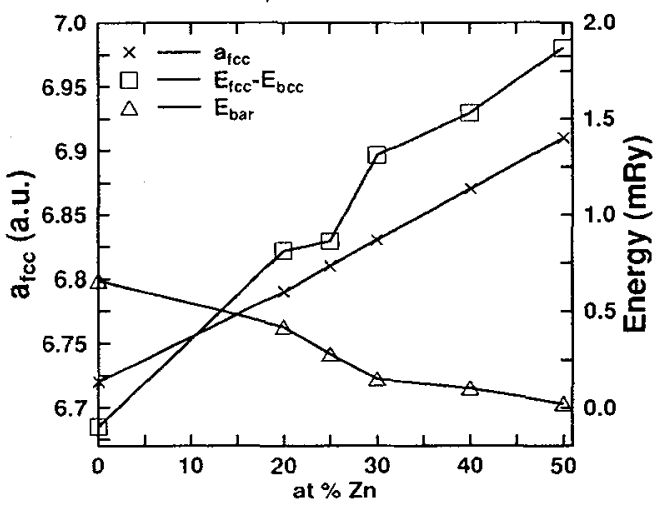

Figure 2 Fcc lattice constants (crosses), energy difference between fcc and bcc state (squares) and energy barriers between fcc and bcc state (triangles) for a) Fe-Ni and b) Cu-Zn. The lines are guides to the cyes.

\subsection{The Cu-Zn System}

The structural binding surfaces for $\mathrm{Cu}_{75} \mathrm{Zn}_{25}$ and for the ordered $\mathrm{Cu}_{3} \mathrm{Zn}$ system are shown in Figure $3 \mathrm{a}$ and $3 \mathrm{~b}$, respectively. The disordered system shows a double minimum structure, the bcc minimum by $\approx 1 \mathrm{mRy}$ more stable than the metastable fcc minimum. The binding surface for $\mathrm{Cu}_{3} \mathrm{Zn}$ looks very peculiar. Although the bcc state is energetically slightly unfavorable, for a large part the system seems indifferent towards a 
change in the c/a ratio. This means that the elastic constant $C^{\prime}$ is very small or possibly negative on a large part of the surface.

Further results for the $\mathrm{Cu}-\mathrm{Zn}$ system are condensed into Figure 2b. Compared to the Fe-Ni system the energy differences between $f c c$ and bcc are very small, which is very plausible since for this system the $3 d$ band is almost completely filled. Fe and $\mathrm{Ni}$ with partly open d-bands are far more sensitive with respect to symmetry changes. In the case of the KKR-CPA the fcc symmetry is only for very low $\mathrm{Zn}$ concentrations given as the ground state, but the fcc state is separated from the bcc by an energy barrier which becomes smaller with increasing Zn amount. The ASW calculations give more bias to the fcc state, the energy difference between fcc and bcc state changing sign at the stoichiometric concentration of $25 \% \mathrm{Zn}$. Among other groundstate properties the lattice constant of the fcc state within the frame of a KKR-CPA calculation has been discussed before.[14] Since in order to obtain the whole binding surface we slightly had to adjust the muffin-tin radius for our present calculation, we find reassurance from the fact, that the lattice constants we find deviate hardly from those obtained by Johnson et. al. [14] and that we still get the same deviation from Vegard's rule.

Preliminary analysis of the valence band filling shows that, like in the Fe-Ni system, besides the $s$ and $d$ band, also the $\mathrm{p}$ band is involved. Since the $\mathrm{d}$ band is almost completely filled, the $\mathrm{s}$ and $\mathrm{p}$ band are filled faster than in the Fe-Ni system, this tendency being even stronger for the $\mathrm{p}$ band than for the $s$ band. The increasing influence of the $\mathrm{p}$ bands could account for the preference of the bcc state with increasing $\mathrm{Zn}$ concentration.
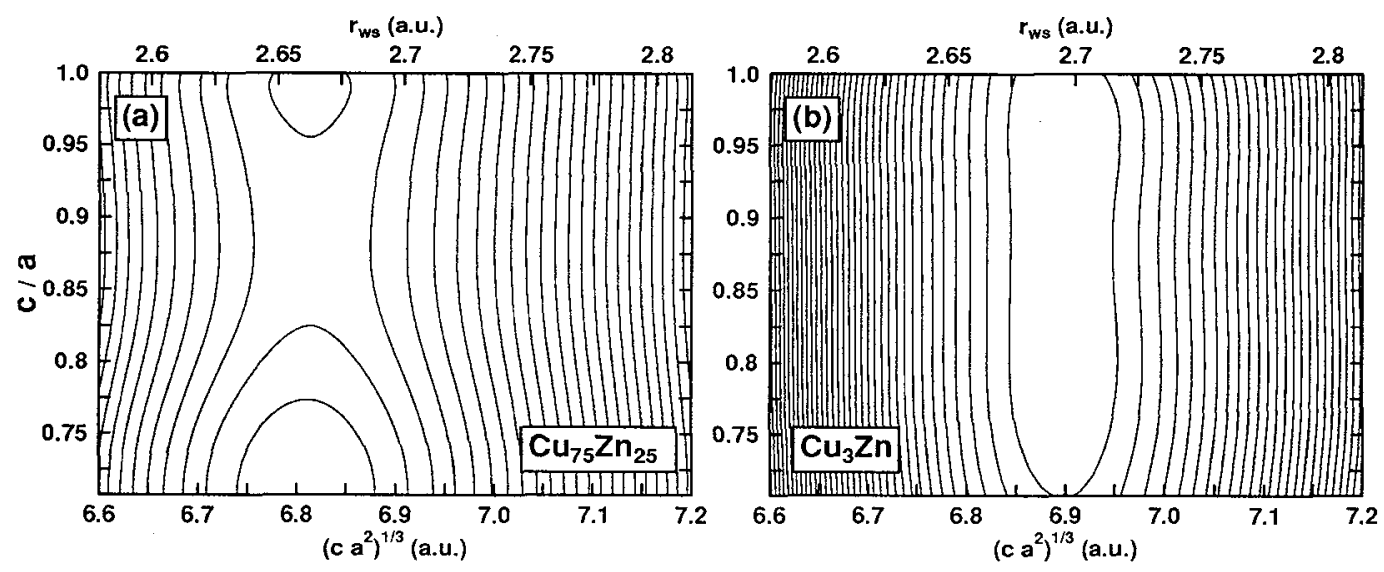

Figure 3 Total energy contours for a) disordered $\mathrm{Cu}_{75} \mathrm{Zn}_{25}$ and b) ordered $\mathrm{Cu} 3 \mathrm{Zn}$. Again the width of the contour lines is in $0.5 \mathrm{mRy}$. As energy differences are small, compared to the Fe-Ni system, a higher resolution could seem appropriate, but the limited accuracy of our calculation would blur the information.

\section{$3.3 \mathrm{Cu}_{50} \mathrm{Zn}_{25} \mathrm{Al}_{25}$}

Replacing half of the amount of zinc with aluminum with respect to $\mathrm{Cu}_{50} \mathrm{Zn}_{50}$ makes the fcc state the stable one, leaving only a metastable minimum close to the bcc c/a ratio. The fact that the fcc state is by $1.1 \mathrm{mRy}$ $(\approx 170 \mathrm{~K})$ more stable than the bcc state and that there is a tiny energy barrier between the two states, fits astonishingly well to the experimental findings of a martensitic transformation temperatures of about the $150 \mathrm{~K} .[15]$

One might be tempted to speculate about the structure halfway between bcc and fcc structure, considering that there are other paths than the Bain path between the two structures. But one has to keep in mind that this structure might be sensitive towards deviation of a tenth of a $m R y$, which is at the extreme limit of resolution of our method. Still a more systematic investigation for similar systems seems necessary in order to obtain more detailed information. Also very inportant and to be considered in the near future, is the 
temperature evolution of these binding surfaces as well as the temperature dependencies of the elastic constants.
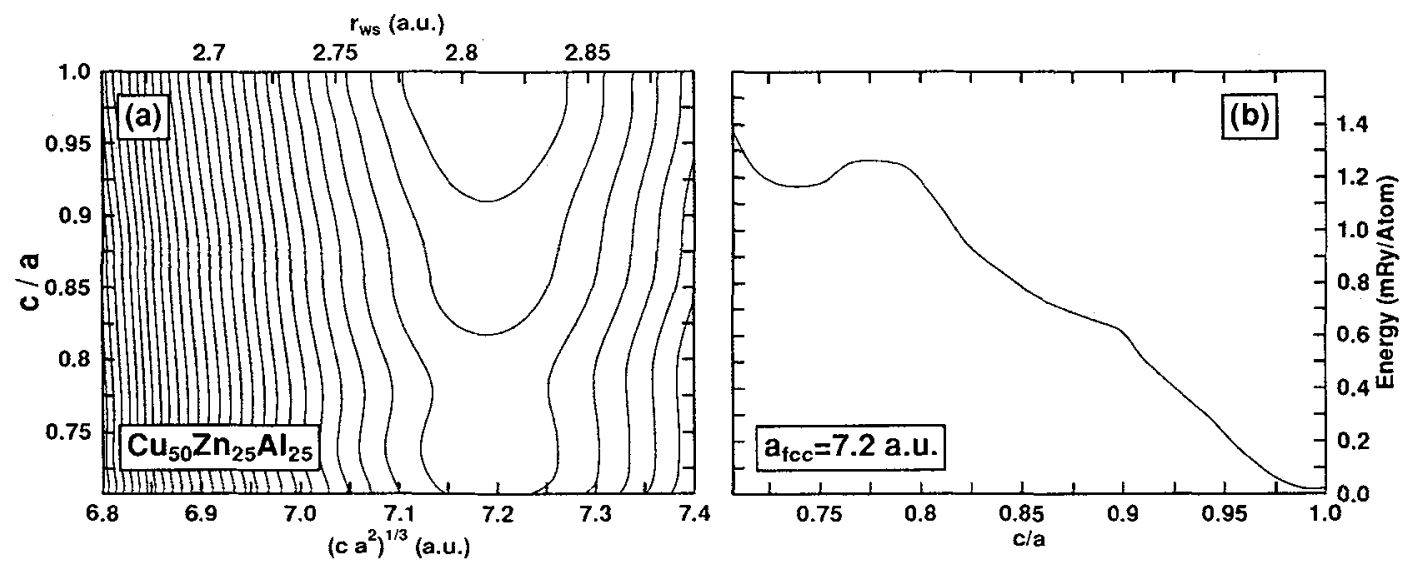

Figure 4 a) total energy contour and b) cross section of the binding surface at constant volume for $\mathrm{Cu}_{50} \mathrm{Zn}_{25} \mathrm{Al}_{25}$. The cross section resolves the energy barrier between the metastable bcc state and the stable fcc state. The structure halfway between fcc and bcc state does not prove to be a third minimum.

\section{Conclusion}

Our analysis shows how magnetism in Fe-Ni systems makes its impact on the structural stability of these systems. At about $50 \% \mathrm{Fe}$ we find that magnetism stabilizes the bcc structure. At about $65 \% \mathrm{Fe}$ the magnetism in the fcc phase breaks down and thus the magnetic phase transition may induce a structural phase transition. For the $\mathrm{Cu}-\mathrm{Zn}$ system we find first indications that the partly filling of $\mathrm{p}$ bands may be responsible for the instability of the fcc state with increasing $\mathrm{Zn}$ concentration. Therefore it will be necessary to calculate the KKR-CPA spectral function (which is the energy and momentum resolved band structure) as well as the Fermi surface, in order to see in more details the destabilizing feature of the $p$ electrons with decreasing $\mathrm{Zn}$ concentration in the Hume-Rothery alloy $\mathrm{Cu}_{50} \mathrm{Zn}_{25} \mathrm{Al}_{25}$.

\section{References}

[1] Kohn W. and Sham L., Phys. Rev 140 (1965) A1133.

[2] Moruzzi V.L., Janak J.F. and Williams A.R., Calculated Electronic Properties of Metals (Pergamon, New York, 1978);

Roy D.M. and Pettifor D.G., J. Phys. F7 (1977) L183.

[3] Schwarz K. and Mohn P., J. Phys. F14 (1984) L129;

Sliwko V.L., Mohn P., Schwarz K. and Blaha P., Phys. Rev. B39 (1989) 3047.

[4] Akai H., J. Phys. Condens. Matter 1 (1989) 8045.

[5] Entel P. and Schröter M., J Physique C8 (1988) 293;

Entel P. and Schröter M., Physica B161 (19889) 121;

Wagner D., J. Phys. Condens. Matter 1 (1989) 4635;

Schröter M., Entel P. and Mishra S.G., J. Magn. Magn. Mat. 87 (1990) 163;

Mohn P., Schwarz K. and Wagner D., Phys. Rev. B43 (1991) 3318.

[6] Williams A.R., Kübler J. and Gelatt C.D., Phys. Rev. B19 (1979) 6094.

[7] von Barth U. and Hedin L., J. Phys. C 5 (1972) 1629.

[8] Janak F., Solid State Commun. 55 (1978) 53.

[9] Entel P., Hoffmann E, Mohn P., Schwarz K and Moruzzi V.L., Phys. Rev B47 (1993) 8706; Hoffmann E., Entel P., Wassermann E., Schwarz K. and Mohn P., "Electronic Structure and Magnetovolume Instabilities of the Hexagonal Laves Phase Compound $\mathrm{Fe}_{2} \mathrm{Ti}^{\prime \prime}$, ESOMAT 94 , 
Barcelona 14-16 September 1994, A. Planes, J. Ortín and L. Mañosa Eds. ( Les Editions de Physique, France, 1995) pp. 129-134.

[10] Korringa J., Physica 13 (1947) 392;

Kohn W. and Rostocker N., Phys. Rev. 94 (1954) 1111.

[11] Shiba H., Prog. Theor. Phys. 46 (1971) 77;

Soven P., Phys. Rev. B2 (1970) 4715.

[12] Johnson D:D. and Pinski F.J., J. Appl. Phys. 57 (1985) 3018;

Johnson D.D., Nicholson D.M., Pinski F.J., Györffy B.L. and Stocks G.M., Phys. Rev. Letters 56 (1986) 2088.

[13] Schröter M., Ebert H., Akai H.,Entel P., Hoffmann E.and Reddy G.G., Phys. Rev. B 52 (1995), 188.

[14] Johnson D.D., Nicholson D.M., Pinski F.J., Györffy B.L. and Stocks G.M., Phys. Rev. B41 (1990) 9701.

[15] Rubini S., Dimitropulos C., Gotthardt R. and Borsa F., Phys. Rev. B44 (1991) 2019. 